\title{
Erratum: Slepton non-universality in the flavor-effective MSSM
}

\section{Luisa López-Ibáñez, ${ }^{a}$ Aurora Melis, ${ }^{a}$ M. Jay Pérez ${ }^{a, b}$ and Oscar Vives ${ }^{a}$}

${ }^{a}$ Departament de Física Tèorica, Universitat de València and IFIC, Universitat de València-CSIC, Dr. Moliner 50, E-46100 Burjassot (València), Spain

${ }^{b}$ Valencia State College, Osceola Campus, 1800 Denn John Ln, Kissimmee, FL, U.S.A.

E-mail: m.luisa.lopez-ibanez@uv.es, aurora.melis@ific.uv.es, mperez75@valenciacollege.edu, oscar.vives@uv.es

ERRATUM TO: JHEP11(2017)162

ARXIV EPRINT: 1710.02593

We report an error in the soft mass matrices due to the values of the flavons in the $\Delta(27)$ model taken as an example in our work [1] (section 3). In this model, we took the VEVs of the flavons to reproduce the observed Yukawa hierarchy as, $v_{3} / M_{\ell}=\sqrt{\alpha} \sim \mathcal{O}(1)$, $v_{23} / M_{\ell} \sim \varepsilon, v_{123} / M_{\ell} \sim \varepsilon^{2}$ and $v_{1} \sim v_{3}$, with the tau yukawa $y_{\tau}$ taken as an overall factor. However, in order to take $y_{\tau}$ out the Yukawa matrix, the correct values of the VEVs are $v_{3} / M_{\ell}=\sqrt{y_{\tau}}, v_{23}=\sqrt{y_{\tau}} \varepsilon$ and $v_{123}=\sqrt{y_{\tau}} \varepsilon^{2}$, as pointed out in ref. [2]. While this has been implicitly taken into account in writing the Yukawa and in the Trilinear matrices, it has not been correctly included in the Kähler and, consequently, in the Soft-Mass matrix.

On the other hand, the factor $f$ in eq. (2.5) of [1] should be corrected as

$$
f=\left(2 N_{\text {in }}-1\right) \cdot\left(2 N_{\text {out }}-1\right)+1 .
$$

Given these considerations, we have to correct eq. (3.11) and then (3.13) of ref. [1] as,

$$
\begin{aligned}
& B_{\ell, R} \rightarrow \alpha\left(\begin{array}{ccc}
2 \varepsilon^{4} & -3(4+8 \alpha) \varepsilon^{3} & 3(4+8 \alpha) \varepsilon^{3} \\
-3(4+8 \alpha) \varepsilon^{3} & 2 \varepsilon^{2} & -2 \varepsilon^{2} \\
3(4+8 \alpha) \varepsilon^{3} & -2 \varepsilon^{2} & 2
\end{array}\right), \\
& m_{\ell, R}^{2} \rightarrow m_{0}^{2}\left(\begin{array}{ccc}
1 & -3 \alpha(3+7 \alpha) \varepsilon^{3} & 3 \alpha\left(3+\frac{11}{2} \alpha-\frac{x_{2}}{3 x_{4}}\right) \varepsilon^{3} \\
-3 \alpha(3+7 \alpha) \varepsilon^{3} & 1+\alpha \varepsilon^{2} & -\alpha\left(1-3 x_{4}\right) \varepsilon^{2} \\
3 \alpha\left(3+\frac{11}{2} \alpha-\frac{x_{2}}{3 x_{4}}\right) \varepsilon^{3}-\alpha\left(1-3 x_{4}\right) \varepsilon^{2} & 1+\alpha
\end{array}\right),
\end{aligned}
$$




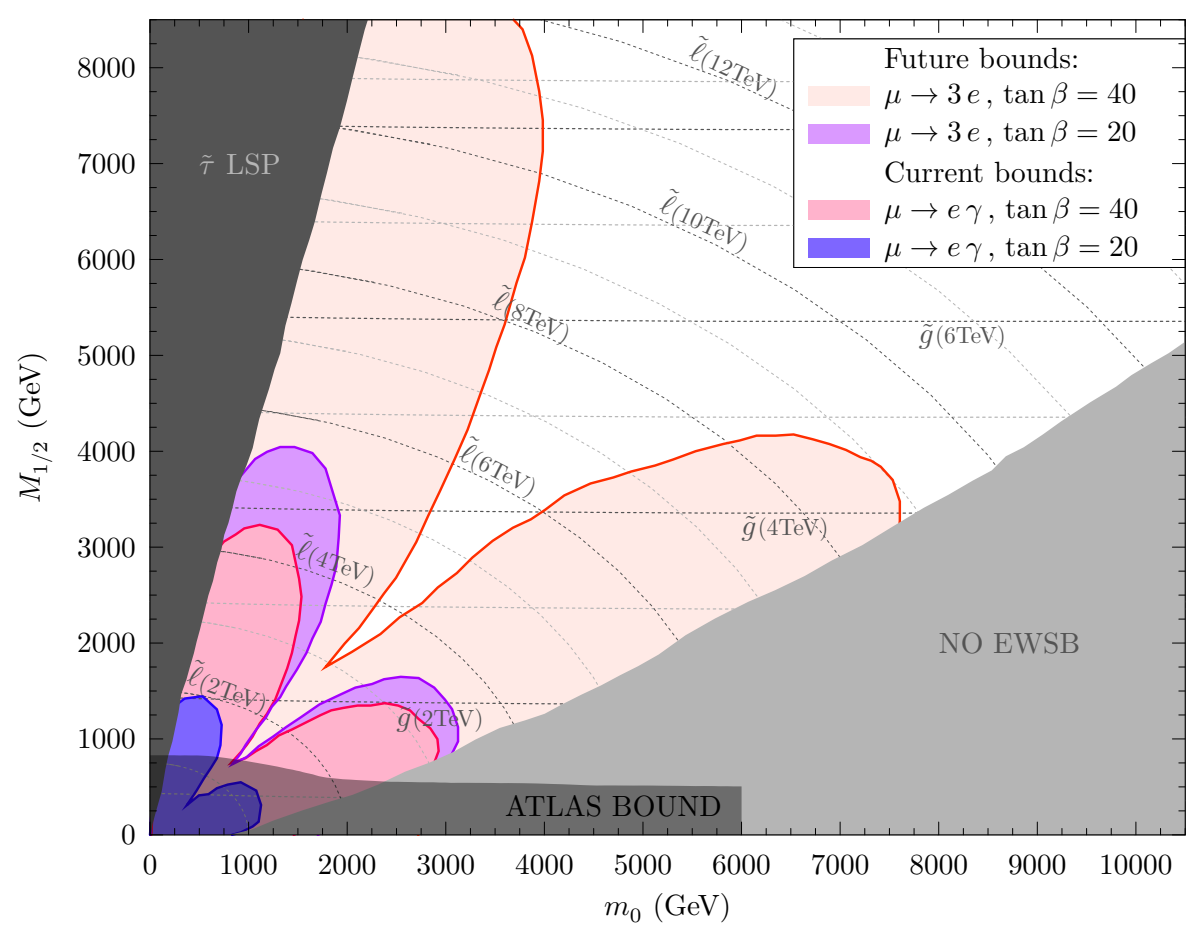

Figure 1. Excluded regions due to $\mu \rightarrow e \gamma$ and $\mu \rightarrow e e e$ for two reference values: $\tan \beta=20$ (blue shapes) and $\tan \beta=40$ (red shapes). In the dark (blue and red) regions, we compare with current $\mu \rightarrow e \gamma$ bounds, while in the light (blue and red) regions we compare with the expected $\mu \rightarrow e e e$ sensitivity in the near future. Interestingly, even for present bounds, these results are competitive with mSUGRA ATLAS limits (gray area).

where $\alpha=y_{\tau}$. Compared to the result of [1], this is an overall $y_{\tau}$ rescaling of the nonuniversal entries that are responsibles of the observed flavor violating effects, which are then significantly reduced. As shown in figure 1, the net effect is a reduction of the excluded parameter space corresponding to this model. Note that, in this figure, we have chosen larger values of $\tan \beta=20,40$, as $\tan \beta=5$ is now giving no interesting constraints. In this figure, we present also the region excluded because of unsuccessful electoweak symmetry breaking, which was not included in the previous figure. A similar region is understood to be excluded also in figures 7 and 8 of [1].

Because of the change in the factor $f$, also the $3 / 2$ factors in eqs. (4.8), (5.8) should be replaced by 2 . However, this change has no impact on the results of ref. [1].

Open Access. This article is distributed under the terms of the Creative Commons Attribution License (CC-BY 4.0), which permits any use, distribution and reproduction in any medium, provided the original author(s) and source are credited.

\section{References}

[1] M.L. López-Ibáñez, A. Melis, M.J. Pérez and O. Vives, Slepton Non-Universality in the Flavor-Effective MSSM, JHEP 11 (2017) 162 [arXiv:1710.02593] [INSPIRE].

[2] I. de Medeiros Varzielas, G.G. Ross and J. Talbert, A Unified Model of Quarks and Leptons with a Universal Texture Zero, JHEP 03 (2018) 007 [arXiv: 1710.01741] [INSPIRE]. 